\title{
Contrast enhanced ultrasound of renal masses. A reappraisal of EFSUMB recommendations and possible emerging applications.
}

\author{
Zeno Sparchez ${ }^{1,2}$, Pompilia Radu², Mihaela Sparchez ${ }^{1,3}$, Nicolae Crisan ${ }^{1,4}$, Gabriel Kacso ${ }^{1,5}$, \\ Bogdan Petrut ${ }^{1,5}$
}

1"Iuliu Hatieganu" University of Medicine and Pharmacy, ${ }^{2}$ Department of Gastroenterology; Regional Institute of Gastroenterology and Hepatology, ${ }^{3}$ Department of Pediatrics, ${ }^{4}$ Urology Department, Cluj-Napoca County Hospital, ${ }^{5}$ Oncological Institute, Cluj-Napoca, Romania.

\begin{abstract}
The main imagistic method for characterization of renal lesions is contrast enhanced computed tomography (CECT). Disadvantages of CECT are a contrast-induced nephropathy in patients with renal impairment, allergic reactions and high costs. Contrast-enhanced ultrasound (CEUS) evaluation of hepatic and non-hepatic lesions is a relatively new, but increasingly utilised, diagnostic method. In 2011 the European Federation of Societies of Ultrasound in Medicine and Biology (EFSUMB) updated the Guidelines and Recommendations on the Clinical Practice of CEUS and included in the recommendation the renal pathology. However, there are several possible new indications that have not been discussed (pyelocaliceal masses and renal vein thrombosis) and several issues that remain controversial such as the differentiation of benign and malignant tumours or the differentiation of lymphoma and metastasis.

This study aims to review literature data, as well as reveal the latest findings in the field of renal CEUS.

Keywords: renal tumours, CECT contrast enhanced computed tomography, CEUS contrast-enhanced ultrasonography.
\end{abstract}

\section{Introduction}

In the recent years the prevalence of renal tumours has been increased, most probably due to an increased detection by using new imaging methods. Nephrectomy or nephron-sparing partial nephrectomy is performed for these renal tumours, but as many as $30 \%$ of the lesions prove to be benign, while another $25 \%$ are low-grade malignant tumours, which generally have less metastatic potential than clear cell renal carcinoma. For these reasons, preoperative assessment of renal mass subtype becomes an important step in diagnosis and therefore the aim of numerous studies. Contrast-enhanced ultrasound (CEUS) evaluation of hepatic and non-hepatic lesions

Received 16.04.2015 Accepted 03.05.2015

Med Ultrason

2015, Vol. 17, No 2, 219-226

Corresponding author: Pompilia Radu

Regional Institute for Gastroenterology

and Hepatology

Croitorilor 19-21

400162, Cluj-Napoca, Romania

E-mail: pia-radu@yahoo.com is a relatively new, but increasingly utilised, diagnostic method.

In 2011 the European Federation of Societies of Ultrasound in Medicine and Biology (EFSUMB) updated the Guidelines and Recommendations on the Clinical Practice of CEUS and recommended several indications for the administration of US contrast agents for the study of several pathology of the kidneys [1]. It has been suggested that CEUS should be considered in cases of renal ischemia, renal infections, renal trauma, for the differentiation of solid renal masses and pseudo tumours, and for the characterization of complex cystic masses. However, there are new evidences that may help in the differentiation of benign and malignant renal tumours and of primary from metastatic lesions. Some new indications have emerged such as the use of CEUS in the diagnosis of pyelocaliceal masses, venous thrombosis in renal cell carcinoma (RCC) and as guidance method in percutaneous biopsy.

This study aims to review literature data, as well as to reveal the latest findings in the field of CEUS use in renal masses. 


\section{EFSUMB recommendations}

\section{Characterisation of complex cystic renal masses}

Renal cysts are common findings seen at US examination. The main goal is to differentiate between a benign and a malignant cyst. To estimate the chance for malignancy, the renal complex cysts are classified according to the Bosniak classification into five categories based on their CT imaging appearance [2]. Unfortunately some of the features of malignancy (e.g. increased echogenicity, calcifications, intracystic septa, or wall thickening) may be also the expression of a complicated benign cyst (e.g. haemorrhage, inflammation, or ischemia) [3]. The most important elements that guide the diagnosis towards the malignity are represented by enhanced thick intracystic septa and enhanced mural nodules after microbubble injection $[4,5]$. Due to the partial volume effect these important clues may be masked by $\mathrm{CT}$, which can lead to a misdiagnosis. According to several reports, CEUS can improve the diagnostic accuracy of both complex cystic lesions detected by US and indeterminate cystic lesions identified on CT or MRI (fig 1) [4-6]. Furthermore several authors showed that CEUS, in non-conclusive renal nodules detected on CT, could differentiate benign complex cysts from other lesions that would require further investigation $[7,8]$.

\section{Differential diagnosis between malignant tumours and pseudotumours}

Pseudo-tumours are developmental masses (columns of Bertin, fetal lobation, dromedary hump) with no pathological significance. This type of tumour can mimic a mass on B mode US exam.

On CEUS exam, these pseudo-lesions follow the exact normal enhancement pattern of the renal cortex or the pyramid, and can thus be differentiated from a true renal mass lesion (fig 2). In 2010, a retrospective study conducted by Mazziotti et al showed that CEUS has similar results to $\mathrm{CT}$ and MRI in establishing the diagnosis of a renal pseudotumour [9].

\section{Renal infections (pyelonephritis or renal abscess)}

The diagnosis of uncomplicated pyelonephritis is based on clinical and laboratory findings. Additional investigations (CEUS or CECT) should be considered if the patient remains febrile after 72 hours of treatment [1].

On CEUS exam, the area of focal acute pyelonephritis (APN) appears as a hypo-enhanced area due to the presence of oedema. Mitterberger et al concluded that CEUS allows small renal parenchymal changes in APN to be detected with high specificity (98\%), sensitivity $(100 \%)$ and moreover, without radiation exposure [10]. In several cases (diabetic or immunosuppressed patients) pyelonephritis can be complicated with parenchymal ab-

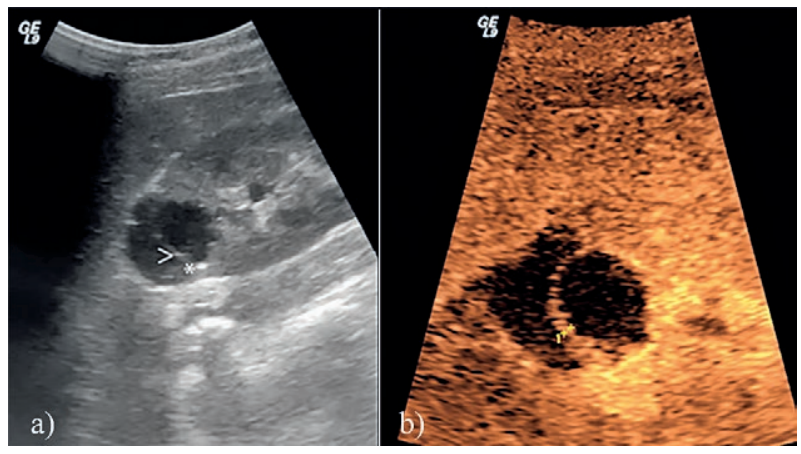

Fig 1. A 33 years old female with a Bosniak $2 \mathrm{~F}$ renal cyst indeterminate on CT: a) US aspect showing a $27 \mathrm{~mm}$ large cyst at the upper pole with intracystic septa $(>)$ and one calcification $(*)$; b) CEUS aspect showing several septae inside the cyst.

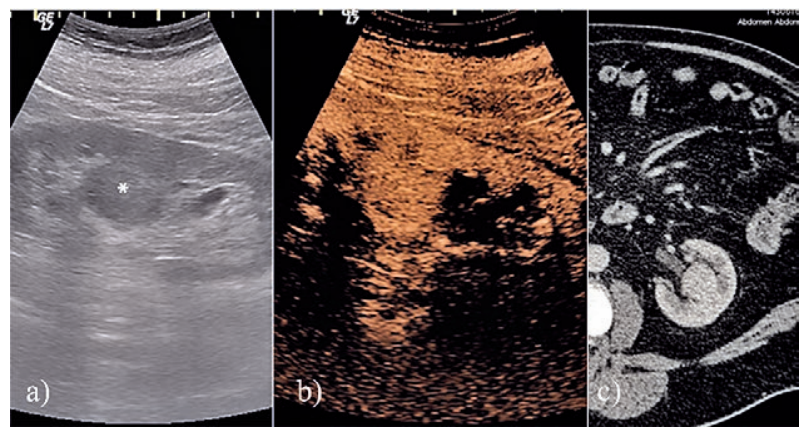

Fig 2. A 74-year old man with hypertrophied column of Bertin: a) coronal B mode US shows a $36 \mathrm{~mm}$ large nodule renal isoechoic with the renal parenchyma $(*)$ protruding in the sinus fat; b) CEUS scan in the cortical phase (12 sec after contrast agent administration) depicts the same enhancement as the renal cortical tissue; c) CT scan in the corticomedullary phase confirm the diagnosis of pseudotumor.

scess formation. The abscess appears as non-enhancing roundish lesions with peripheral uptake (fig 3). For these patients the use of CEUS was also recommended for the follow-up [11].

The puss in the collecting system or bladder has no vessels, therefore it shows no enhancement, being easily differentiated from neoplastic tissues [5].

\section{Renal ischemia}

In the case of suspected renal ischemia, colour and power Doppler US are the first imaging modalities to identify focal perfusion defects.

On CEUS examination, the renal infarcts appear as triangular or wedge shaped areas with no contrast uptake, while the rest of the parenchyma enhances normally. Moreover, CEUS allows the differential diagnosis between infarction and cortical ischemia, where enhancement of segmental, interlobular and arciform vessels is recognized, while enhancement of interlobular vessels of the renal cortex is lacking [12]. 


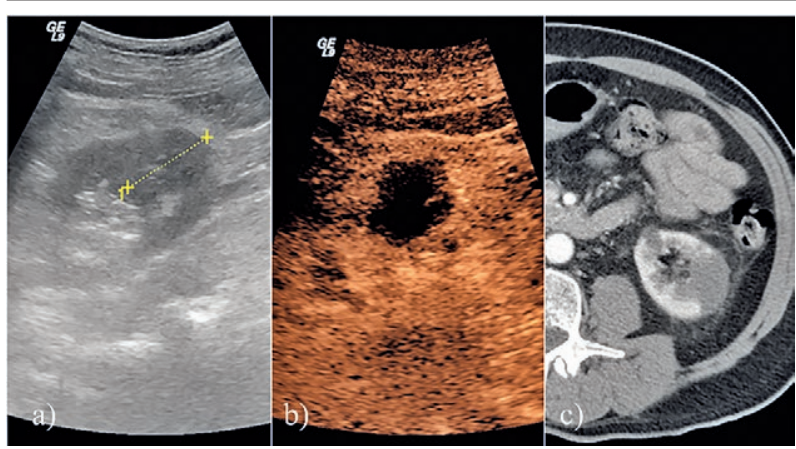

Fig 3. A 65-year old man with a renal lesion after a urinary infection treated with antibiotics: a) US shows a $3.8 \mathrm{~cm}$ isoechoic lesion at the lower pole; b) CEUS in cortical phase (12 sec after injection) shows no enhancement in the lesion but a slightly hyperenhancement around; c) CT scan shows no enhancement in the arterial phase.

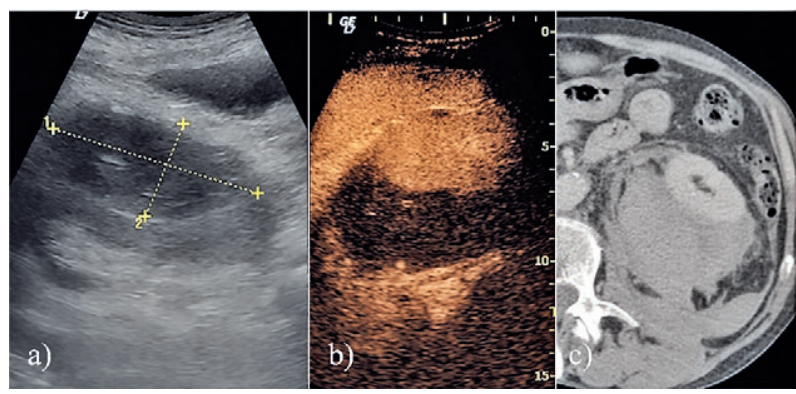

Fig 4. A 75 year old man with a large renal lesion after abdominal trauma: a) US shows a $86 / 40 \mathrm{~mm}$ hypoechoic around the right kidney; b) CEUS revealed no enhancement in the lesion; c) CT shows lack of enhancement in the lesion, certifying the diagnosis of perirenal haematoma.

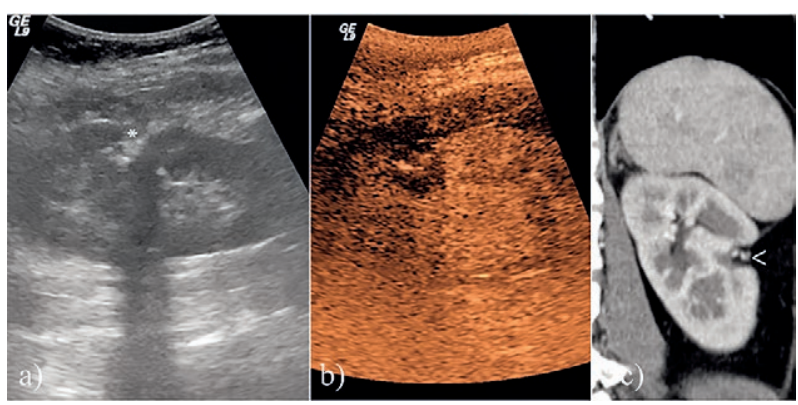

Fig 5. A 43 female patient with RCC after intraoperative RFA; a) US shows a hypoechoic area with retraction of the capsula $(*) ; b)$ CEUS revealed no enhancement in the corticomedullary phase; $\mathrm{c}$ ) CT depicted a non-enhanced area compatibile with necrosis $(<)$.

Another important application of CEUS in patients with renal ischemia is the differentiation between nonperfused, infarcted tissue and hypoperfused parenchymal regions. On Doppler US both appear as areas lacking colour signal, but on CEUS examination, only infracted areas are completely non-enhanced.

\section{Renal Trauma}

The first-line imaging method in emergency room of severe traumatic patients is the conventional FAST (Focus Assessment with Sonography in Trauma) US. FAST-US can detect free abdominal, pleural, and pericardial fluids, but it has low sensitivity in the detection of parenchymal (liver, kidneys, and spleen) traumatic lesions, which may be isoechoic and misdiagnosed. The contusions appear as slightly hypoechogenic areas with blurred margins, lacerations as anechogenic streaks or bands that reach the surface of the organ and ischemic changes as well-defined avascular areas. Moreover, in up to one third of the number of cases, parenchimal organ injuries may be present without haemoperitoneum [13]. Due to the limitations of US (inability for deep breath, poor imaging of gut perforation, or pancreatic trauma) and to the ability of CECT to offer an overview of the whole body, CECT is the reference examination and modality of choice for a patient with multiple abdominal injuries. However, CECT has several disadvantages such as radiation, time, money, and false negative results that decrease the value of this method.

In several cases, such as localized injuries, CEUS can be of great value, revealing abdominal solid organ lesions not visible on baseline US [14]. On CEUS examination, traumatic renal and perirenal lacerations and haematomas appear as non-enhancing areas (fig 4) [13]. The major limit of CEUS compared with CECT remains the impossibility of identifying lesions of the collecting system.

\section{Monitoring of renal lesions treated by percutaneous ablation therapies}

Local treatment (i.e. radiofrequency ablation-RFA, microwave ablation-MWA, cryoablation) is minimally invasive and allows for a fast recovery. Therefore many urologists and radiologists prefer these techniques. Clinical success for the treatment of RCCs requires a complete destruction of the tumour, therefore a good imaging tool for evaluating the tumour after ablation is necessary. CEUS has proved to be a useful tool for the assessment of the treatment (fig 5) [15]. In a series of patients with renal tumours treated by RFA in whom a prevalence of residual disease of $19 \%$ was documented, the sensitivity and specificity of CEUS in early detection of residual tumours was $64 \%$ and $98 \%$, respectively [16].

Moreover CEUS allows a „real-time” monitorization of the ablated area during RFA procedure which may provide a higher success rate than in the case of US monitoring [17]. These results were confirmed by another study where CEUS was superior to clasic US monitoring in achieving the local tumour control (100\% vs. $87.5 \%)$. Despite the fact that the difference did not reach statisti- 
cal significance, it is obvious that CEUS has the potential to provide more effective renal tumour ablation [18]. In a very recent study it was demonstrated that CEUS has an excellent agreement with $\mathrm{CT}$ and a high sensitivity in the follow-up of patients with small renal masses treated with cryosurgery, demonstrating its usefulness in assessing the efficiency of ablation [19].

\section{Controversial and undiscussed issues.}

\section{Differential diagnosis between benign and malignant tumours.}

The treatment strategies and prognosis for malignant and benign tumours is quite different; thus differential diagnosis is crucial. The goal of imaging renal tumours is therefore to differentiate malignant from benign tumours. Even if US is the first imaging tool for diagnosis, it has a low sensitivity in differentiating between a malignant lesions (e.g. RCCs) from benign lesions (e.g. angiomyolipomas-AMLs, oncocytomas). The current methods used to establish the right diagnosis of renal masses are CECT and magnetic resonance imaging (MRI) [20].

The main differential diagnosis involves the distinction between the minimal fat renal AMLs, oncocytomas, and RCCs [21,22]. Minimal fat AMLs includes two entities, the epithelioid (EAML) and triphasic minimal fat AML (TAML). It is important to underline that the EAML has a malignant potential and requires surgical treatment, just like RCCs [23].

On CEUS and CECT minimal fat AMLs (both TAML and EAML types), show a centripetal enhancement in corticomedullary phase with homogeneous peak enhancement followed by iso-enhancement in parenchymal phase $[24,25]$. Oncocytomas are seen on CEUS exam as earlier spoke-like hyperenhancement, fast washout compared to the surrounding renal cortex, a nonenhanced rim, and central irregular nonenhanced areas [22].

The heterogeneous enhancement, a perilesional rim (pseudocapsula) and hypoenhancement in the late cortico-medullary and venous phase are the main characteristic traits of RCCs on CEUS or CECT exam (fig 6) [24-26].

Several authors showed that the tumour size influences the homogeneity of enhancement [26,27]. Taking into account that RCCs $<3 \mathrm{~cm}$ and AMLs have both a homogeneous enhancement pattern, it was suggested that CEUS is capable to differentiate malignant from benign lesions only in tumours $>3 \mathrm{~cm}$ [27].

Histology alongside size is another factor that may influence the behaviour of RCCs on CEUS exam [28]. In cortical phase, clear cell RCCs display usually homogeneous hyper-enhancement, papillary RCCs less hy-

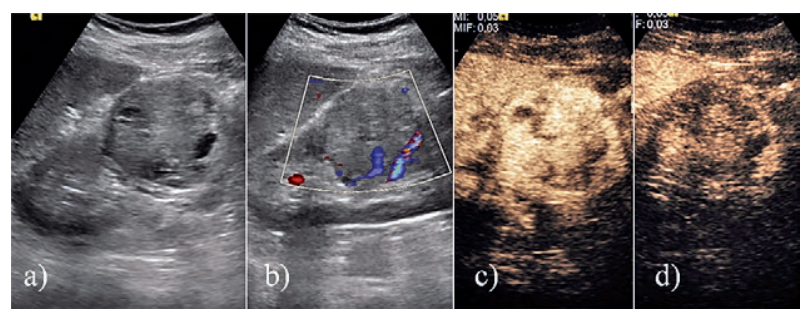

Fig 6. A 44 years old man with clear cell RCC; a) US shows a $4.8 \mathrm{~cm}$ renal inhomogeneous tumor with cystic areas inside; b) on color Doppler US the lesion was well vascularized; c) on CEUS scan, in corticomedullay phase, the tumour enhanced earlier and more intense that the surrounding parenchyma; d) in venous phase the tumour became hypoenhanced.

per- and heterogeneous enhancement, and chromophobe RCC heterogeneous iso-enhancement. Compared to the rapid wash-in and rapid washout pattern of clear RCC, papillary and chromophobe RCCs exhibited the slow wash-in and rapid washout pattern [28]. According to Li et al [28], the sensitivity, specificity, accuracy, positive predictive value, and negative predictive value of CEUS for the diagnosis of malignant renal lesions are $93.3 \%$, $96.5 \% 96 \%, 82.4 \%$, and $98.8 \%$ for CEUS [28]. The main difficulty is to differentiate oncocytomas from chromophobe RCCs, as they have almost identical CEUS pattern [28]. A recent metaanalysis showed that the pooled sensitivity and specificity of CEUS in the diagnosis of RCC is $88 \%$ and $80 \%$, respectively [20].

The new data from published studies and meta-analysis, in which new tumoral enhancement features (like the timing of wash-in and wash-out) were analysed, have demonstrated that CEUS can be used with good accuracy in the diagnostic of renal tumours [20,27-30]. This is somehow in contradiction with previous studies that have considered CEUS an unreliable imaging method of RCC, due to chaotic vascularization, without typical enhancement patterns [31].

\section{Differential diagnosis between RCCs and other renal malignancy.}

The renal parenchyma can be the place for metastasis of different non-renal solid cancers (e.g. lung, breast, colon, oesophagus, liver, pancreatic, prostate, and thyroid) or lymphomas. In patients diagnosed with lymphoma, a renal involvement has been reported in 34-60\% of cases [32].

The common US findings of lymphoma involvement are: a) multiple hypoechoic masses (usually small 1-3 $\mathrm{cm}$ lesions, without capsula, not totally spheric, sometimes wedge-shaped), b) diffuse renal infiltration (almost always bilateral), and c) renal invasion from contiguous retroperitoneal disease (in approximately $25-30 \%$ of patients) (fig 7). Other US features are absence of a mass effect on renal vessels and pelvicalyceal system 


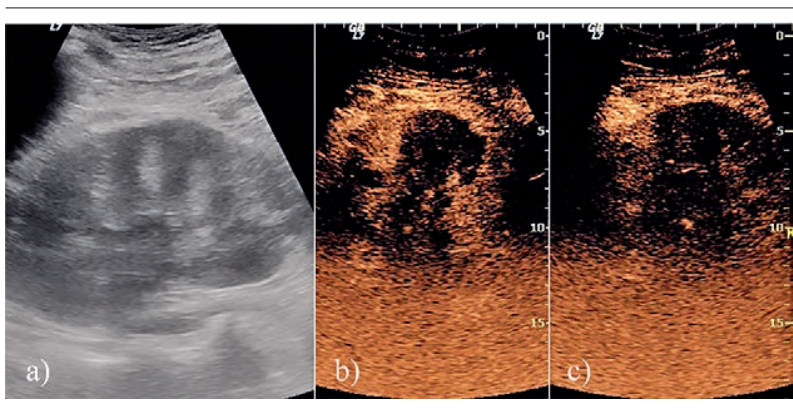

Fig 7. A 77 years old man with retroperitoneal non Hodgkin lymphoma: a) US shows an inhomogeneous right kidney without any obvious mass; b) CEUS in early cortiocomedullary phase $(24 \mathrm{sec})$ depicted an hypoenhanced area in the middle part of the kidey; c) in venous phase, $80 \mathrm{sec}$ after injection, the lesion and the lower pole are marked hypoenhanced.

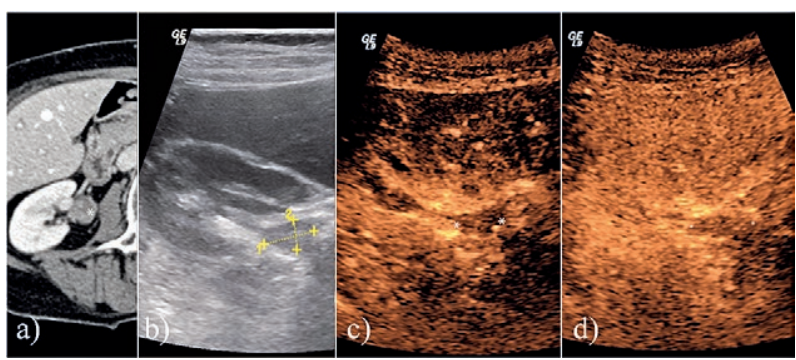

Fig 8. A 63 years old female with hematuria: a) CT shows a mass in the right pielouretheral jonction with suspicion of urothelial carcinoma; b) US shows a $18 \mathrm{~mm}$ hyperechoic mass; on CEUS the lesion was hypoenhanced in both c) early cortical and d) late corticomedullary phase. Surgical specimen confirmed urothelial carcinoma.

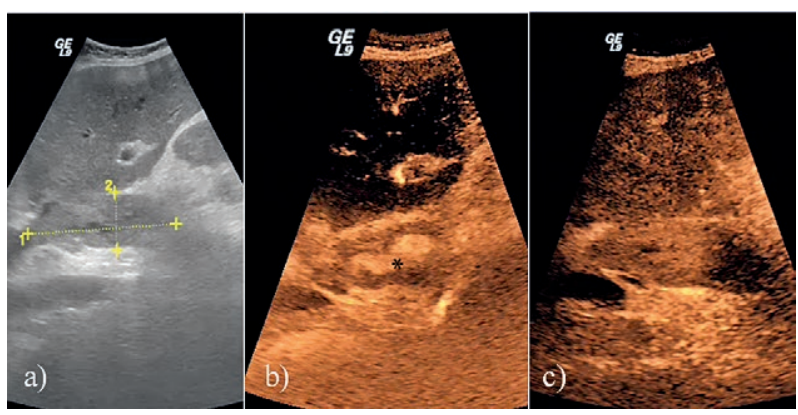

Fig 9. A 73 years old male with RCC of the right kidney and renal vein and inferior vena cava thrombus: a) US shows an echogenic, unhomogenoeous thrombus in the inferior vena cava; b) CEUS in early arterial phase displays a mixt enhancement pattern (hyper-enhanced in the middle * and hypoenhanced at periphery); c) in venous phase the thrombus is hypoenhanced.

and absence of calcification [32,33]. Renal metastases may present as solitary, multiple, or infiltrative masses. In solitary lesions, the differentiation from a RCC is an important clinical issue.

On CEUS exam, both types of metastatic lesions have similar behaviour (hypo or isoenhancement in early corticamedulary phase followed by an early wash- out) $[30,34,35]$. Ignee A et al [30] suggested that non-renal tumour metastases in CEUS exam are typically hypovascular in more than $80 \%$ of cases [30].

As RCCs may display the same pattern, CEUS cannot be used to differentiate RCCs and other renal malignancy $[30,34,35]$.

\section{Establishing the nature of pyelocaliceal masses}

Primary tumours of the renal pelvis and ureter account for up to $10-15 \%$ of all urinary tract tumours, more than $90 \%$ of which are urothelial carcinomas [36]. The role of US in diagnosing the type of intracaliceal /intraurethral masses is limited. On US examination, the urothelial carcinomas are iso- or hypoechoic lesions compared to surrounding renal parenchyma [37]. Also, Doppler US cannot bring more details due to the low vascularity of these tumours. In CEUS, the dynamic enhancement pattern is characterised by a slow-in $(73.5 \%$ of cases), fastout $(94 \%)$ and low enhancement (77.6\%) compared with the renal cortex (fig 8) [38].

The main advantage of CEUS in clinical practice is the possibility to differentiate pyelocalicial tumours from other non-tumoral non-enhancing masses such as clots or organized pus. In case of enhancing masses located mostly in the pelvic uretheral junction the lack of washout or a late wash-out may suggest benign lesions such as uretheral papilloma or fibroepithelial polyps [39].

\section{New insights. Emerging application of CEUS}

\section{Assessment of renal vein and inferior vena cava thrombosis (extent, nature)}

The RCCs have the tendency to extend in the renal veins and the inferior vena cava in $21-35 \%$ and $4-10 \%$ of cases, respectively [40]. An important goal of preoperative imaging is to determine the extension and the nature of the renal thrombus and to select the best surgical approach.

US is the first tool performed to diagnose the presence/ absence of the renal vein or inferior cava vein (IVC) thrombus. The reported sensitivity of detecting IVC thrombosis depends on the localisation of the thrombus, with lower sensitivity for infrahepatic than for intrahepatic thrombi (68\% and $100 \%$ respectively) [41]. Other limitations are the lack of IVC visualisation (in up to $40 \%$ of cases) and the impossibility to distinguish bland from tumoral thrombi [42].

CEUS may overcome this limit demonstrating an enhancement during the corticomedulary phase in tumoral thrombi and the absence of CA in the bland one (fig 9) [30]. It may be also possible to depict the enhancement pattern in the mixed thrombi. CEUS had comparable re- 
sults to CECT in the staging of vein invasion but seems to be superior in the differentiation of bland and tumoral renal vein involvement [30].

Multidetector CT (MDCT) has proved to be superior to the conventional $\mathrm{CT}$ in delineating the extent of tumour thrombus with sensitivity of $93 \%$ and a specificity of $80 \%$ [41]. Currently, with $96-100 \%$ sensitivity, MRI is considered the "gold standard" in detecting both the presence and extension of the thrombus in IVC [41].

New data suggest that tumour thrombus consistency is another important factor for staging [43]. Whether the thrombus consistency may be assessed by CEUS is not known, further studies being necessary.

\section{Quantitative imaging}

This new technique generates time-intensity curves (TICs), which plot echo intensity against time. These TICs are performed for an objective analysis of the tumoural tissue enhancement. TICs are performed for both tumoural tissue (a manually determined region of interest -ROI- selected by the radiologist) and normal renal parenchyma. The enhancement features analyzed in this study included: arrival time (AT), time to peak (TTP), washout time (WT), maximum intensity ( Imax) and intensity at $60 \mathrm{~s}$ (I60).

Dong et al reviewed 42 cases of RCCs using TIC analysis but they failed to find a characteristic perfusion pattern for all RCCs. In spite of this, the authors confirmed the observation that TTP of tumoral parenchyma was shorter than that of normal renal parenchyma [44]. Similar results were obtained by Lu et al [23].

Cai $Y$ et al [45] showed that early washout in the area of maximal intensity inside of the lesion and prolonged washout in whole area of the lesion are suggestive for RCC. This behavoir seems to be the imaging expression of heterogeneous angiogenesis. The authors recommended to combine the hyper-enhancement in the wash-in phase with relative earlier washout of the maximal intensity area in the interior of the lesion to increase the specificity for diagnosing RCC [45].

Based on these encouraging results it seems that this new technique has a great potential for stratifying renal masses into benign and malignant, but further studies are necessary to assess the real value.

\section{Optimisation of percutaneous renal biopsy}

The actual indications for biopsy in renal masses are well documented [46-48], but their presentation are beyond the purpose of our review.

In renal tumours, core biopsy performed with large needles has better performances $(80-100 \%$ overall sensitivity, $97.7 \%$ for malignancy) than fine needles aspiration biopsy but is itself influenced by tumoral size and structure [48]. In case of small renal masses (less than 3-4 cm)

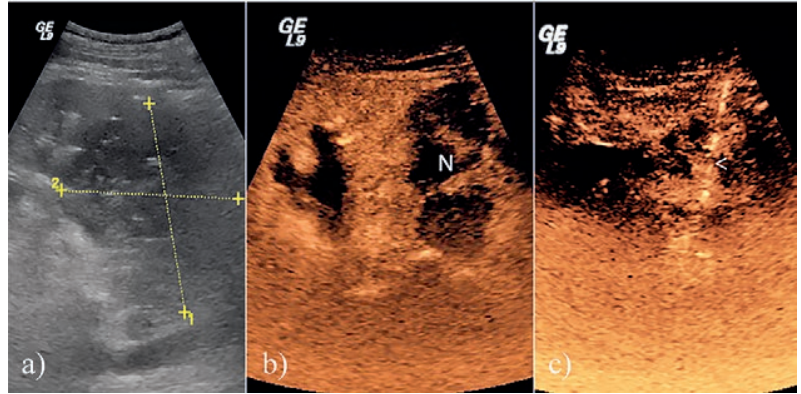

Fig 10. Large renal tumour in a 65 years old female: a) US shows a large, very inhomogeneous tumour at the lower pole; b) in arterial phase the lesion has several necrotic areas, $\mathrm{N}$ - necrosis; c) using CEUS guidance percutaneous biopsy was performed, a specimen being sampled from the enhanced area $(<$ needle).

only $80-84 \%$ of core biopsies were diagnostic in a recent study [49]. For large lesions ( $>6 \mathrm{~cm}$ ), the sensitivity is also relatively low (87\%) with even lower negative predictive value (44\%) [50].

The main factors explaining these results concern the changes occurring during the growth of the tumour (such as intratumoral necrosis, haemorrhage, or cystic degeneration) and misstargeting of small or less visible lesions [50]. In order to surpass these limits, several authors have demonstrated that using CEUS before or during biopsy, non-diagnostic tissue (fibrotic or necrotic) can be avoided. CEUS also increased the conspicuity of less defined / invisible lesions, issue demonstrated for hepatic tumours $[51,52]$. It has been also demonstrated that CEUS accurately depicts the vascularized, active parts in partially ablated renal tumours [16].

In a study performed by our group that included 28 patients with large renal lesions, it was demonstrated that CEUS guided biopsy is a feasible technique that can be performed both in arterial or early venous phase. Owing to the differentiation of necrotic from viable tissue in the tumour and to a better depiction of less visible or diffuse lesions (especially in renal lymphoma) the sensitivity reached $100 \%$ (fig 10) [53]. The authors recommended that in case of large tumours with necrotic or cystic intratumoral areas or tumours poorly visible on conventional US, the biopsy should be always performed with CEUS guidance $[53,54]$.

\section{Conclusions}

CEUS is a safe, accurate alternative capable of improving the diagnostic capabilities of B mode US. It provides a new imagistic tool to investigate patients when CT/MRI imaging is either contraindicated or yielding equivocal finding. Guidelines recommend CEUS to: a) 
characterize cystic masses; b) differentiate diagnosis renal tumours and pseudo-tumours when B mode US is equivocal; c) diagnose renal abscesses in clinically-suspected patients with impaired renal function, and d) replace the standard CECT/ MRI in the follow up after focal ablation or surgery, with a subunitary cost effective ratio. New application may emerge such as the quantitative analysis of enhancement features of various tumours, to help the differentiation diagnosis between malignant and benign tumours, the characterisation of venous thrombi in RCC helpful for correct surgical planning and improvement of percutaneous guided biopsy.

\section{References}

1. Piscaglia F, Nolsře C, Dietrich CF, et al. The EFSUMB guidelines and recommendations on the clinical practice of contrast enhanced ultrasound (CEUS): update 2011 on non-hepatic applications. Ultraschall Med 2012; 33: 3359.

2. Bosniak MA. The Bosniak renal cyst classification: 25 years later. Radiology 2012; 262: 781-785.

3. Nicolau C, Bunesch L, Sebastia C. Renal complex cysts in adults: contrast-enhanced ultrasound. Abdom Imaging 2011; 36: 742-752.

4. Xue LY, Lu Q, Huang BJ, et al. Contrast-enhanced ultrasonography for evaluation of cystic renal mass: in comparison to contrast-enhanced CT and conventional ultrasound. Abdom Imaging 2014; 39: 1274-1283.

5. Cokkinos DD, Antypa EG, Skilakaki M, Kriketou D, Tavernaraki E, Piperopoulos PN. Contrast enhanced ultrasound of the kidneys: what is it capable of? Biomed Res Int 2013; 2013: 595873 .

6. Clevert DA, Minaifar N, Weckbach S, et al. Multislice computed tomography versus contrast-enhanced ultrasound in evaluation of complex cystic renal masses using the Bosniak classification system. Clin Hemorheol Microcirc 2008; 39: 171-178.

7. Barr RG, Peterson C, Hindi A. Evaluation of indeterminate renal masses with contrast-enhanced US: a diagnostic performance study. Radiology 2014; 271: 133-142.

8. Nicolau C, Buñesch L, Paño B, et al. Prospective evaluation of CT indeterminate renal masses using US and contrastenhanced ultrasound. Abdom Imaging 2015; 40: 542-551.

9. Mazziotti S, Zimbaro F, Pandolfo A, Racchiusa S, Settineri $\mathrm{N}$, Ascenti G. Usefulness of contrast-enhanced ultrasonography in the diagnosis of renal pseudotumors. Abdom Imaging 2010; 35: 241-245.

10. Mitterberger M, Pinggera GM, Colleselli D, et al. Acute pyelonephritis: comparison of diagnosis with computed tomography and contrast-enhanced ultrasonography. BJU Int 2008; 101: 341-344.

11. Correas JM, Claudon M, Tranquart F, Hélénon AO. The kidney: imaging with microbubble contrast agents. Ultrasound Q. 2006; 22: 53-66.
12. Bertolotto M, Catalano O. Contrast-enhanced Ultrasound: Past, Present, and Future. Ultrasound Clinics 2009; 4: 339367.

13. Cokkinos DD, Antypa E, Kalogeropoulos I, et al. Contrastenhanced ultrasound performed under urgent conditions. Indications, review of the technique, clinical examples and limitations. Insights Imaging 2013; 4: 185-198.

14. Valentino M, Ansaloni L, Catena F, Pavlica P, Pinna AD, Barozzi L. Contrast-enhanced ultrasonography in blunt abdominal trauma: considerations after 5 years of experience. Radiol Med 2009; 114: 1080-1093.

15. Salvaggio G, Campisi A, Lo Greco V, Cannella I, Meloni MF, Caruso G. Evaluation of post treatment response of hepatocellular carcinoma: comparison of ultrasonography with second-generation ultrasound contrast agent and multidetector CT. Abdom Imaging 2010; 35: 447-453.

16. Hoeffel C, Pousset M, Timsit MO, et al. Radiofrequency ablation of renal tumours: diagnostic accuracy of contrastenhanced ultrasound for early detection of residual tumour. Eur Radiol 2010;, 20: 1812-1821

17. Zhao X, Wang W, Zhang S, at al. Improved outcome of percutaneous radiofrequency ablation in renal cell carcinoma: a retrospective study of intraoperative contrast-enhanced ultrasonography in 73 patients. Abdom Imaging 2012; 37: 885-891.

18. Chen Y, Huang J, Xia L, et al. Monitoring laparoscopic radiofrequency renal lesions in real time using contrast-enhanced ultrasonography: an open-label, randomized, comparative pilot trial. J Endourol 2013; 27: 697-704.

19. Sanz E, Hevia V, Arias F, et al. Contrast-enhanced ultrasound (CEUS): an excellent tool in the follow-up of small renal masses treated with cryoablation. Curr Urol Rep 2015; 16: 469.

20. Wang C, Yu C, Yang F, Yang G. Diagnostic accuracy of contrast-enhanced ultrasound for renal cell carcinoma: a meta-analysis. Tumour Biol 2014; 35: 6343-6350.

21. Pallwein-Prettner L, Flöry D, Rotter CR, et al. Assessment and characterisation of common renal masses with $\mathrm{CT}$ and MRI. Insights Imaging 2011; 2: 543-556.

22. Wu Y, Du L, Li F, Zhang H, Cai Y, Jia X. Renal oncocytoma: contrast-enhanced sonographic features. J Ultrasound Med 2013; 32: 441-448.

23. Lu Q, Li CX, Huang BJ, Xue LY, Wang WP. Triphasic and epithelioid minimal fat renal angiomyolipoma and clear cell renal cell carcinoma: qualitative and quantitative CEUS characteristics and distinguishing features. Abdom Imaging 2015; 40: 333-342.

24. Xu ZF, Xu HX, Xie XY, Liu GJ, Zheng YL, Lu MD. Renal cell carcinoma and renal angiomyolipoma: differential diagnosis with real-time contrast-enhanced ultrasonography. J Ultrasound Med 2010; 29: 709-717.

25. Lu Q, Wang W, Huang B, Li C, Li C. Minimal fat renal angiomyolipoma: the initial study with contrast-enhanced ultrasonography. Ultrasound Med Biol 2012; 38: 1896-1901.

26. Xu ZF, Xu HX, Xie XY, et al. Renal cell carcinoma: realtime contrast-enhanced ultrasound findings. Abdom Imaging 2010; 35: 750-756. 
27. Jiang J, Chen Y, Zhou Y, Zhang H. Clear cell renal cell carcinoma: contrast-enhanced ultrasound features relation to tumor size. Eur J Radiol 2010; 73: 162-167.

28. Li X, Liang P, Guo M, et al. Real-time contrast-enhanced ultrasound in diagnosis of solid renal lesions. Discov Med 2013; 16: 15-25.

29. Zhou X, Yan F, Luo Y, et al. Characterization and diagnostic confidence of contrast-enhanced ultrasound for solid renal tumors. Ultrasound Med Biol 2011; 37: 845-853.

30. Ignee A, Straub B, Brix D, Schuessler G, Ott M, Dietrich $\mathrm{CF}$. The value of contrast enhanced ultrasound (CEUS) in the characterization of patients with renal masses. Clin Hemorheol Microcirc 2010; 46: 275-290.

31. Haendl T, Strobel D, Legal W, Frieser M, Hahn EG, Bernatik T. Renal cell cancer does not show a typical perfusion pattern in contrast-enhanced ultrasound. Ultraschall Med 2009; 30: 58-63.

32. El-Sharkawy MS, Siddiqui N, Aleem A, Diab AA. Renal involvement in lymphoma: prevalence and various patterns of involvement on abdominal CT. Int Urol Nephrol 2007; 39: 929-933.

33. Zhang J, Lefkowitz RA, Bach A. Imaging of kidney cancer. Radiol Clin North Am 2007; 45: 119-147.

34. Trenker C, Neesse A, Görg C. Sonographic patterns of renal lymphoma in B-mode imaging and in contrast-enhanced ultrasound (CEUS)-A retrospective evaluation. Eur J Radiol 2015; 84: 807-810.

35. Gulati M, King KG, Gill IS, Pham V, Grant E, Duddalwar VA. Contrast-enhanced ultrasound (CEUS) of cystic and solid renal lesions: a review. Abdom Imaging 2015 Jan 15. doi: 10.1007/s00261-015-0348-5.

36. Prando A, Prando P, Prando D. Urothelial cancer of the renal pelvicaliceal system: unusual imaging manifestations. Radiographics 2010; 30: 1553-1566.

37. Browne RF, Meehan CP, Colville J, Power R, Torreggiani WC. Transitional cell carcinoma of the upper urinary tract: spectrum of imaging findings. Radiographics 2005; 25: 1609-1627.

38. Xue LY, Lu Q, Huang BJ, et al. Evaluation of renal urothelial carcinoma by contrast-enhanced ultrasonography. Eur J Radiol. 2013; 82: e151-e157.

39. Choi YH, Kim SH, Cho JY, Kim SH. Unusual fibroepithelial polyp in renal pelvis. Abdom Imaging 2008; 33: 98-100.

40. Davits RJ, Blom JH, Schrhder FH. Surgical management of renal carcinoma with extensive involvement of the vena cava and right atrium. Br J Urol 1992: 70: 591-593.

41. Guo HF, Song Y, Na YQ. Value of abdominal ultrasound scan, CT and MRI for diagnosing inferior vena cava tumour thrombus in renal cell carcinoma. Chin Med J (Engl) 2009; 122: $2299-2302$.
42. Trombetta C, Liguori G, Bucci S, Benvenuto S, Garaffa G, Belgrano E. Evaluation of tumor thrombi in the inferior vena cava with intraoperative ultrasound. World J Urol 2007; 25: 381-384.

43. Bertini R, Roscigno M, Freschi M, et al. Impact of venous tumour thrombus consistency (solid vs friable) on cancerspecific survival in patients with renal cell carcinoma. Eur Urol 2011; 60: 358-365.

44. Dong XQ, Shen Y, Xu LW, Xu CM, Bi W, Wang XM. Contrast-enhanced ultrasound for detection and diagnosis of renal clear cell carcinoma. Chin Med J 2009; 122: 11791183.

45. Cai Y, Du L, Li F, Gu J, Bai M. Quantification of enhancement of renal parenchymal masses with contrast-enhanced ultrasound. Ultrasound Med Biol 2014; 40: 1387-1393.

46. Sahni VA, Silverman SG. Imaging management of incidentally detected small renal masses. Semin Intervent Radiol 2014; 3: 9-19.

47. Halverson SJ, Kunju LP, Bhalla R, et al. Accuracy of determining small renal mass management with risk stratified biopsies: confirmation by final pathology. J Urol 2013; 189 : 441-446.

48. Maturen KE, Nghiem HV, Caoili EM, Higgins EG, Wolf JS Jr, Wood DP Jr. Renal mass core biopsy: accuracy and impact on clinical management. AJR Am J Roentgenol 2007; 188: $563-570$.

49. Leveridge MJ, Finelli A, Kachura JR, et al. Outcomes of small renal mass needle core biopsy, nondiagnostic percutaneous biopsy, and the role of repeat biopsy. Eur Urol 2011; 60: 578-584.

50. Rybicki FJ, Shu KM, Cibas ES, Fielding JR, vanSonnenberg E, Silverman SG. Percutaneous biopsy of renal masses: sensitivity and negative predictive value stratified by clinical setting and size of masses. AJR Am J Roentgenol 2003; 180: 1281-1277.

51. Grossjohann HS, Bachmann Nielsen. Ultrasound contrast agents may help in avoiding necrotic areas at biopsy. Ultraschall Med 2006; 27: 2-3.

52. Schlottmann K, Klebl F, Zorger N, Feuerbach S, Schölmerich J. CEUS allows for interventions of hepatic lesions which are invisible on B mode. Z Gastroenterol 2004; 42: 303-310.

53. Sparchez Z, Radu P, Kacso G, et al. Performance of CEUS guided biopsy in large renal and adrenal tumors. Ultrasound Med Biol 2011; 37: S33.

54. Spârchez Z, Radu P, Zaharia T, Kacso G, Grigorescu I, Badea R. Contrast enhanced ultrasound guidance: a new tool to improve accuracy in percutaneous biopsies. Med Ultrason 2010; 12: 133-138. 